\title{
Influence of adhesive rough surface contact on microswitches
}

\author{
Ling Wu, ${ }^{1,2, a)}$ V. Rochus, ${ }^{1}$ L. Noels, ${ }^{1}$ and J. C. Golinval ${ }^{1}$ \\ ${ }^{1}$ Department of Aerospace and Mechanical Engineering, University of Liege, 1, Chemin des Chevreuils, \\ Liège 4000, Belgium \\ ${ }^{2}$ School of Aeronautics, Northwestern Polytechnical University, Xi'an 710072 China
}

(Received 27 July 2009; accepted 17 October 2009; published online 1 December 2009)

\begin{abstract}
Stiction is a major failure mode in microelectromechanical systems (MEMS). Undesirable stiction, which results from contact between surfaces, threatens the reliability of MEMS severely as it breaks the actuation function of MEMS switches, for example. Although it may be possible to avoid stiction by increasing restoring forces using high spring constants, it follows that the actuation voltage has also to be increased significantly, which reduces the efficiency. In our research, an electrostatic-structural analysis is performed to estimate the proper design range of the equivalent spring constant, which is the main factor of restoring force in MEMS switches. The upper limit of equivalent spring constant is evaluated based on the initial gap width, the dielectric thickness, and the expected actuation voltage. The lower limit is assessed on the value of adhesive forces between the two contacting rough surfaces. The MEMS devices studied here are assumed to work in a dry environment. In these operating conditions only the van der Waals forces have to be considered for adhesion. A statistical model is used to simulate the rough surface, and the Maugis's model is combined with Kim's expansion to calculate adhesive forces. In the resulting model, the critical value of the spring stiffness depends on the material and surface properties, such as the elastic modulus, surface energy, and surface roughness. The aim of this research is to propose simple rules for design purposes. (C) 2009 American Institute of Physics. [doi:10.1063/1.3260248]
\end{abstract}

\section{INTRODUCTION}

Electrostatic actuated devices are the most common microelectromechanical systems (MEMS) devices due to their simplicity, as they require few mechanical components and small voltage levels for actuation. This kind of MEMS devices is susceptible to an instability that is known as pull-in: ${ }^{1,2}$ when the applied voltage is increased beyond a critical value, which is called the pull-in voltage, the movable electrode will collapse onto the fixed electrode. As the bias voltage decreases, the strong adhesion of the contact point may keep the two conducting electrodes sticking together instead of letting the moving one go back to its initial position, which results in a major failure of the device.

Due to the microscopic scale, surface forces are more important than volume forces, and stiction becomes a typical failure mode of MEMS. ${ }^{3-5}$ Stiction is the effect that microscopic structures tend to adhere to each other when their surfaces come into contact and when the restoring forces are unable to overcome the interfacial forces. As generating artificial forces on MEMS is difficult to achieve, it is often impossible to separate the adhered surfaces again. Since incidental contact cannot be excluded completely, and since contact between moving parts can be the purpose of some kinds of MEMS (e.g., switches), this problem influences the design and functionality of micro- and nanodevices. In order to overcome the adhesion forces generated at the contact interface, enough restoring forces should be provided by the deformed structure. Although increasing equivalent spring constant can increase restoring forces, the actuation voltage

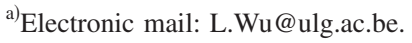

will increase significantly as a result, which reduces the device efficiency. Hence, the design parameters should be properly selected based on the knowledge of adhesive forces.

The most important adhesive forces or surface forces are capillary condensation, ${ }^{6,7}$ molecular van der Waals forces, electrostatic forces, $\mathrm{H}$ bridging, and solid bridging. $\mathrm{H}$ bridging can only be observable for hydrophilic, $\mathrm{OH}$ terminated surfaces, in a waterless environment, while the last two can be neglected compared with the other forces. ${ }^{8}$ Capillary adhesion could occur during the final fabrication stages involving the chemical wet etch and liquid rinse (release stiction), during the normal operation at high humidity environments (in-use stiction), or while parts are stored (dormancy stiction). In a dry and low-pressure environment, capillary forces will be relatively small, and the dominative factor of in-use stiction is the van der Waals forces between the molecules. ${ }^{8,9}$

The use of atomic force microscopes (AFMs) and of surface force apparatuses, which allow for the accurate measurement of surface forces when the dimensions of contacting bodies are small, has shown that surface roughness plays an important role in the magnitude of the pull-out force between two bodies. The characterization of surface roughness is challenging because asperity distribution on the surface is not uniform. As an example, Fig. $1^{10}$ shows a representative AFM surface image of an electrode. ${ }^{10}$ The Greenwood and Williamson's (GW) asperity model ${ }^{11}$ is the pioneer model developed to account for the contact between the rough surfaces. The contact problem is statistically studied based on three assumptions: (1) all asperities on a rough surface have the same radius of curvature, (2) asperity height follows a Gaussian distribution, and (3) asperities are independent of each other. Hence, the three parameters of the GW model are 


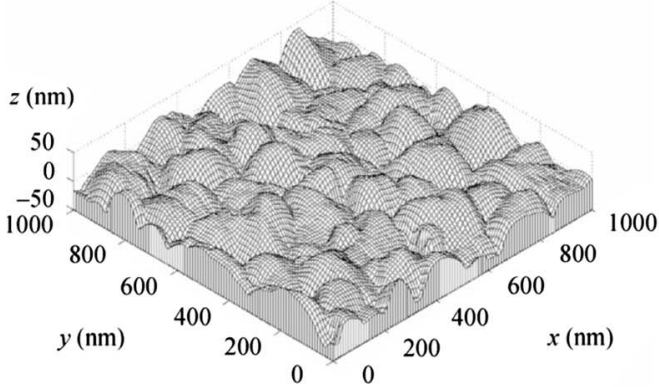

(a)

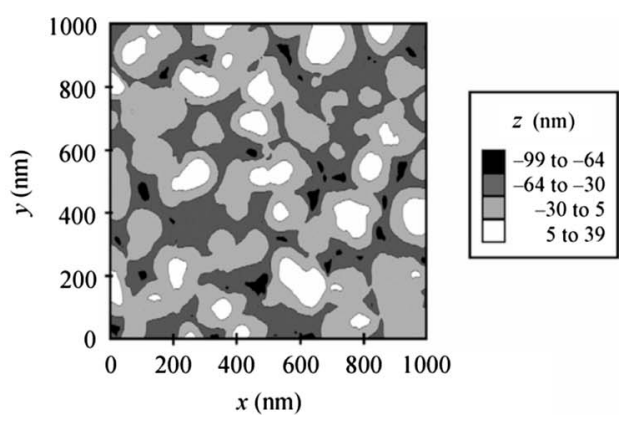

(b)

FIG. 1. (a) AFM surface topography image. (b) 2D contour plot of the surface of a side electrode. Reprinted with permission from A. Lumbantobing, L. Kogut, K. Komvopoulos, J. Microelectromech. Syst. 13, 977 (2004). Copyright @ 2004, IEEE.

the constant radius of the spherical asperities $R$, the standard deviation of the asperity height $\sigma$ [referred to as root-meansquare (rms) roughness], and the asperity density $N$. The value of these characterizing parameters of rough surfaces can be determined from AFM images. ${ }^{10}$

Adhesive contact between elastic bodies has been modeled by Johnson, Kendall, and Roberts (JKR), ${ }^{12}$ by Derjaguin, Muller, and Toporov (DMT) ${ }^{13}$ and by Maugis, who proposed the so-called transition solution. ${ }^{14}$ The JKR theory has been the first one to determine the compliance relationships for the contact of elastic spheres in the presence of surface energies. It assumes that the attractive intermolecular surface forces cause elastic deformations beyond that predicted by the Hertz theory, thereby producing a subsequent increase in the contact area. In this model, the surface forces are short-ranged and act only inside the contact area, so this theory is ideal for soft, compliant materials with high surface energy. The DMT theory, in contrast, accounts for the longranged adhesive forces outside the contact area (a ringshaped zone of the noncontact area) but assumes that the contact profile remains the same as in the Hertz theory. This theory is well suited for harder, less compliant materials with low surface energy and small asperity tip radius. By combining contact and fracture mechanics theories, the Maugis theory provides transition solutions for intermediate cases between JKR and DMT regimes. In the present paper, adhesive forces will be modeled by recourse to the Maugis theory, which will be discussed in detail in Sec. II.

In this work, typical kind of electrostatically actuated MEMS devices, such as capacitive MEMS switches, is studied. These capacitive MEMS switches use a thin layer of dielectric material to separate two conducting electrodes; when actuated, this dielectric layer prevents direct metal-tometal contact. Hence, contact results from interactions between the surface asperities and the true contact area is reduced to a very small proportion of the apparent surface contact area. The pull-in and adhesion phenomena of this kind of MEMS device are studied for a uniform surface roughness in Sec. II of this paper. The proper range of equivalent spring constant, which is the main factor of restoring force in MEMS switches for design purposes, is then investigated in Sec. III. As for real surfaces the roughness is not uniform, our model is extended to statistical roughness distribution in Sec. IV. The same study in Sec. III is then conducted in Sec. V, demonstrating that increasing the standard deviation of the asperity height increases the design range. Eventually, a discussion on the parameter identification is provided in Sec. VI.

\section{ELECTROSTATIC-MECHANICAL MODEL}

In the studied one-dimensional (1D) electrostaticmechanical model, asperities on the surfaces are modeled by spheres. For the sake of simplicity, the contact between two spheres is reduced to the contact between an equivalent sphere with a flat plane according to the contact theory, which will be explained in Sec. II C. In order to simplify the problem and pay the main effort on physical phenomena, in this section, the one unit model, which contains only one asperity, as shown in Fig. 2, is applied when studying the impact of adhesion on MEMS devices.

\section{A. Equilibrium equation}

Consider the system sketched in Fig. 2, which consists of a fixed metal plate covered with a dielectric layer, and a movable upper metal plate fastened with a spring. A DC voltage can be applied between the two metal plates in order to activate the switch. The distance $d$ represents the gap between the asperity mean height and the dielectric surface. Due to the definition, when the asperity enters into contact with the dielectric under the action of the voltage, the distance $d$ remains positive. $d_{0}$ is the equilibrium mean separation of the plates for zero DC voltage.

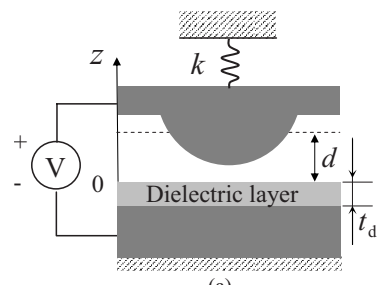

(a)

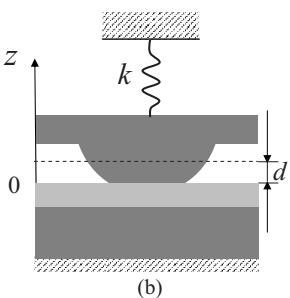

(b)
FIG. 2. Schematic view of one unit of rough surface model. The dashed line represents asperity mean height plane, from which the mean separation between the upper and lower plates is defined: (a) before pull in (not to scale) and (b) after pull in (not to scale). 


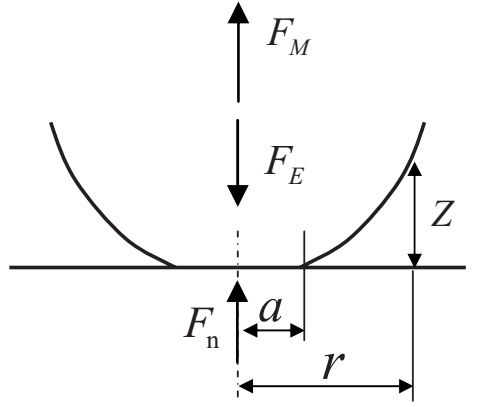

FIG. 3. Schematic of various forces acting on one asperity unit.

Before pull-in [Fig. 2(a)], the forces acting on the upper metal plate are the electrostatic force $F_{E}$ and the mechanical force $F_{M}$ from the spring. The static equilibrium equation of the system is

$$
F_{M}+F_{E}=k\left(d_{0}-d\right)+F_{E}=0
$$

where $k$ is the constant spring stiffness.

After pull-in [Fig. 2(b)], an extra force arises at contact interfaces (see Fig. 3). Also, the static equilibrium equation of the system becomes

$$
F_{M}+F_{E}+F_{n}=0,
$$

where $F_{n}$ is the interaction contact force, which includes the adhesion forces.

\section{B. Electrostatic force}

In the following calculation, the dielectric layer is assumed to be perfectly planar and smooth. ${ }^{15}$ Let us consider one unit of the rough surface which contains only one asperity, as illustrated in Fig. 2.

The sum of electrostatic forces is divided into two parts: the electrostatic force at contact area and electrostatic force at noncontact area. Neglecting the fringing of electrical field, we have

$$
F_{E}=\int_{A_{-} \text {contact }}-\frac{\varepsilon_{d} V^{2}}{2 t_{d}^{2}} \mathrm{~d} A+\int_{A_{-} \text {noncontact }}-\frac{\varepsilon_{0} V^{2}}{2\left(z+\frac{t_{d}}{\varepsilon_{r}}\right)^{2}} \mathrm{~d} A,
$$

where $\varepsilon_{0}$ and $\varepsilon_{\mathrm{d}}$ are the permittivities of air and of the dielectric layer, respectively, $\varepsilon_{r}=\varepsilon_{d} / \varepsilon_{0}$ is the relative permittivity of the dielectric material, $A_{-}$contact is the area in effective contact, and $A_{-}$noncontact is the projected area of the asperity separated by an air spacing $z$ (Fig. 3). For the noncontact position, as show in Fig. 2(a), the electrostatic force at contact area is zero, and Eq. (1) becomes

$$
F_{M}+F_{E}=k\left(d_{0}-d\right)+\int_{A_{-} \text {noncontact }}-\frac{\varepsilon_{0} V^{2}}{2\left(z+\frac{t_{d}}{\varepsilon_{r}}\right)^{2}} \mathrm{~d} A=0,
$$

where $z$ is the local air gap. Expression of the electrostatic force $F_{E}$ in Eq. (4) is justified as the asperity is modeled by a sphere, and as the asperity's deformation due to the elec-

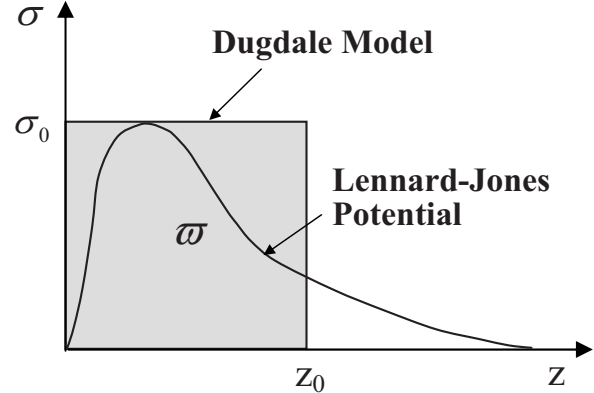

FIG. 4. Dugdale and Lennard-Jones force-separation law.

trostatic force can be neglected compared to the size of air gap before pull in.

\section{Adhesion and contact between spherical surfaces}

Maugis theory is an analytical theory based on a Dugdale assumption, which consists of constant traction within a critical value of separation, for interaction potential: once $z$ exceeds a threshold $z_{0}$, the adhesive traction immediately falls to zero, as shown in Fig. 4. Another way to model the adhesive force is based on the use of a Lennard-Jones potential. Figure 4 shows the comparison between Dugdale model and the more physically based Lennard-Jones potential. Although an obvious difference between these two models can be seen, the Dugdale assumption is accurate enough to describe the contact of spheres, except when large accuracy is required. ${ }^{16}$

In this paper, Dugdale interaction model is considered, and the work of adhesion $\varpi$ is represented by the gray rectangular area in Fig. 4. This work of adhesion $\varpi$ is given by $\varpi=\gamma_{1}+\gamma_{2}-\gamma_{12}$, where $\gamma_{1}$ and $\gamma_{2}$ represent the surface energy of each interacting body, and $\gamma_{12}$ is the interface energy of the two materials. Therefore, the Maugis' transition parameter is defined as ${ }^{17}$

$$
\lambda=\frac{2 \sigma_{0}}{\left(\pi \varpi K^{2} / R\right)^{1 / 3}},
$$

where the effective radius $R$ of two spheres in contact is $R$ $=R_{1} R_{2} /\left(R_{1}+R_{2}\right)$. The reduced elastic modulus $K$ is defined by $K=\frac{4}{3}\left[\left(1-\nu_{1}^{2}\right) / E_{1}+\left(1-\nu_{2}^{2}\right) / E_{2}\right]^{-1}$, where $E_{1}, E_{2}, \nu_{1}$, and $\nu_{2}$ represent the Young's modulus and Poisson's ratio for the respective spheres. $\sigma_{0}$ is the maximum adhesive traction shown in Fig. 4, and it is chosen to match the maximum adhesive traction derived from the Lennard-Jones potential. ${ }^{18}$

The value of $\lambda$ ranges from zero to infinity. For the limiting case of compliant materials with large radii and high adhesive forces, $\lambda=\infty$, and Maugis model is identical to JKR model, which only considers short-ranged interaction forces acting inside the contact area. ${ }^{12}$ For small radii of curvature and low surface adhesive forces, $\lambda=0$, and Maugis model is identical to DMT model, which accounts for the adhesive forces outside of the contact area (a ring-shaped zone of the noncontact). ${ }^{13}$ For in between values of $\lambda$, Maugis model realizes a continuous transition between the JKR and DMT models.

The three governing equations of Maugis' theory for the transition solution of two adhesive spheres in contact are 

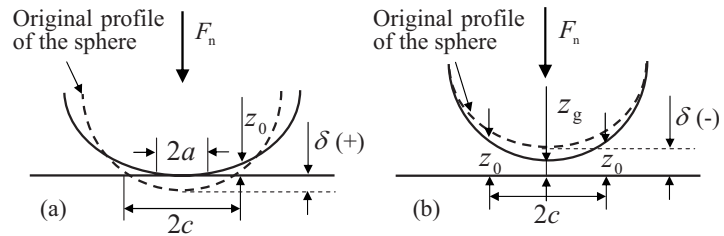

FIG. 5. Illustration of physical parameters. (a) Contact radius $a$ and adhesive contact radius $c$, interference $\delta$ is a positive value. (b) Parameters in Kim et al. extension, interference $\delta$ is a negative value.

$$
\begin{aligned}
1= & \frac{\lambda A^{2}}{2}\left[\sqrt{m^{2}-1}+\left(m^{2}-2\right) \arctan \sqrt{m^{2}-1}\right] \\
& +\frac{4 \lambda^{2} A}{3}\left[\sqrt{m^{2}-1} \arctan \sqrt{m^{2}-1}-m+1\right], \\
\bar{F}_{n}= & A^{3}-\lambda A^{2}\left(\sqrt{m^{2}-1}+m^{2} \arctan \sqrt{m^{2}-1}\right), \\
\Delta & =A^{2}-\frac{4}{3} A \lambda \sqrt{m^{2}-1},
\end{aligned}
$$

where the parameter $m$ is equal to $c / a$. In these relations, $c$, which depends on the value of $z_{0}$ and on the deformation of the sphere, is the adhesive interaction radius, and $a$ is the intimate contact radius [see Fig. 5(a)]. In expression (8), $A$, $\overline{F_{n}}$, and $\Delta$ are the dimensionless contact radius, load (positive if compressive), and approach (interference), respectively, and are defined as follows:

$$
\begin{aligned}
& A=\frac{a}{\left(\pi \varpi R^{2} / K\right)^{1 / 3}}, \\
& \bar{F}_{n}=\frac{F_{n}}{\pi \varpi R}, \\
& \Delta=\frac{\delta}{\left(\pi^{2} \varpi^{2} R / K^{2}\right)^{1 / 3}} .
\end{aligned}
$$

The intimate contact radius $a$, adhesive contact radius $c$, applied load $F_{n}$, and approach (interference) $\delta$ are presented in Fig. 5(a). The approach (interference) $\delta$ is expressed as

$$
\delta=\frac{a^{2}}{R}-\frac{8 \sigma_{0}}{3 K} \sqrt{c^{2}-a^{2}} .
$$

Kim et al. ${ }^{19}$ extended the solution of Maugis-Dugdale to the noncontact regime of $a=0$ and $c \neq 0$ [see Fig. 5(b)] by the adjustment of Eqs. (6)-(8), leading to

$$
\begin{aligned}
& \frac{\pi}{4} C^{2} \lambda+\frac{2}{3}(\pi-2) C \lambda^{2}+\xi=1, \\
& \bar{F}_{n}=-\frac{\pi}{2} C^{2} \lambda, \\
& \Delta=-\frac{4}{3} C \lambda-\frac{2}{\pi} \frac{\xi}{\lambda},
\end{aligned}
$$

where $C=c /\left(\pi \varpi R^{2} / K\right)^{1 / 3}$ represents the dimensionless adhesive contact radius, and $\xi=z_{g} / z_{0}$ [see Fig. 5(b)].

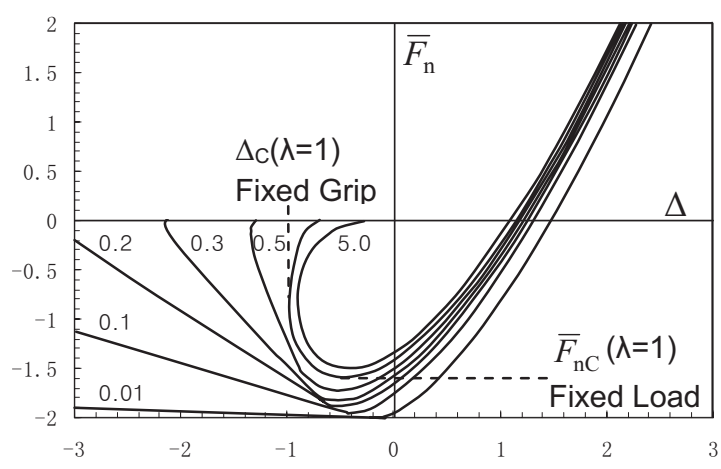

FIG. 6. Dimensionless load approach curves for adhesive contact of two spherical surfaces.

Resolution of Eqs. (6)-(8) and (13)-(15) yields the determination of the load, interference, and contact radius. Figure 6 shows the load versus deflection curve for different values of $\lambda$. In particular, the pull-out, which consists of the lost of contact can be deduced from this picture: (i) for each value of the transition parameter $\lambda(5)$, the horizontal tangent represents the tensile force at which abrupt pull-out occurs under load control, and (ii) in displacement control conditions, the vertical tangent is the location for the abrupt pullout, $\Delta_{C}$. Such vertical tangent exists only if $\lambda>0.938$, in which case Maugis solution is correct as pull-out occurs for an intimate contact [Fig. 5(a)]. On the other hand, if $\lambda$ $<0.938$ pull-out occurs for a noncontact configuration [Fig. $5(\mathrm{~b})]$, in which case the Kim et al. ${ }^{19}$ correction is required.

Based on the models recalled here above, we are now able to compute the force balances for different configurations of a given idealized MEMS device. In particular, the pull-in voltage and the pull-out voltage or the stiction configuration can be predicted.

\section{DESIGN FOR UNIFORM ROUGHNESS}

In this section, the adhesion models described previously are exploited in order to design the spring constant so that pull-in phenomenon occurs for a reasonable actuation voltage, and stiction risk is avoided. In order to extract easily the effect of each phenomenon, a surface made of uniform asperities is considered. This will be extended to realistic rough surfaces in Sec. IV.

\section{A. Pull in}

From Eq. (4), we can get the pull-in voltage, which is the maximum voltage with respect to the air gap $d$,

$$
V_{\text {pull in }}=\max \left\{\left[\frac{k\left(d_{0}-d\right)}{\int_{A_{-} \text {noncontact }} \frac{\varepsilon_{0}}{2\left(z+\frac{t_{d}}{\varepsilon_{r}}\right)^{2}} \mathrm{~d} A}\right]^{1 / 2}\right\}
$$

If length quantities $d$ and $z$ are normalized with respect to $d_{0}$, leading to, respectively, $\bar{d}$ and $\bar{z}$, if $\bar{k}=k / A_{a}$, where $A_{a}$ is equal to the area of the electrode plate, and if $\bar{t}_{d}$ $=t_{d} /\left(d_{0} \varepsilon_{r}\right)$, Eq. (16) can be rewritten as 

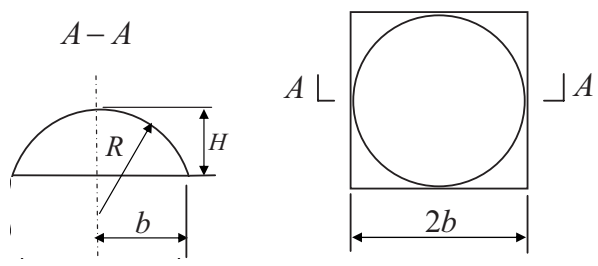

FIG. 7. Schematic of the extreme unit.

$$
\begin{aligned}
& V_{\text {pull in }} \\
& =\left(\frac{d_{0}^{3}}{\varepsilon_{0}}\right)^{1 / 2} \max \left\{\left[\frac{\bar{k}(1-\bar{d})}{\frac{1}{A_{a}} \int_{A_{-} \text {noncontact }} \frac{1}{2\left(\bar{z}+\bar{t}_{d}\right)^{2}} \mathrm{~d} A}\right]^{1 / 2}\right\} .
\end{aligned}
$$

The influence of the asperity on the pull-in voltage is investigated with an extreme unit model, in which the asperity occupies the whole unit (see Fig. 7). The extreme unit model indicates that the asperities are arranged side by side on the surface.

If the asperities are neglected, the pull-in voltage of a flat plate with equilibrium mean separation at zero DC voltage $d_{0}$ is obtained from Eq. (17) and is equal to

$$
V_{\text {pull in }}=\eta \sqrt{\bar{k}} \text { and } \eta=\left[\frac{8 d_{0}^{3}\left(1+\bar{t}_{d}\right)^{3}}{27 \varepsilon_{0}}\right]^{1 / 2},
$$

where the coefficient $\eta$ represents the integral effect of initial gap size, dielectric layer thickness, and permittivity of the pull-in voltage. The effect of asperities can be studied by normalizing the exact pull-in voltage (17) by the approximation (18), leading to the normalized pull-in voltage $\bar{V}_{\text {pull in }}$, which is equal to 1 in the absence of asperities.

The typical geometric and electrostatic parameters of MEMS devices are used to study the effect of asperities on pull-in voltage. With $d_{0}=2 \mu \mathrm{m}, t_{d}=0.2 \mu \mathrm{m}$, and $\varepsilon_{r}=4$, the normalized pull in-voltage $\bar{V}_{\text {pull in }}$ versus normalized asperity height $H / R$ for different radii of asperity is plotted in Fig. 8. These curves have been obtained by numerically solving Eq. (17). We observe that the pull-in voltage increases with the asperity height increase. This results from the fact that $d_{0}$ considered in Eq. (18) is the distance from the smooth plate to the mean asperity height (Fig. 2). Therefore, when the

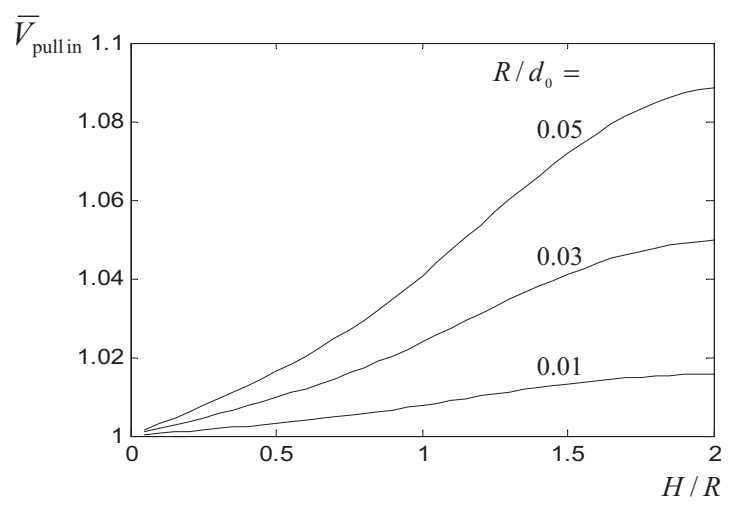

FIG. 8. Effect of asperity height on the pull-in voltage.

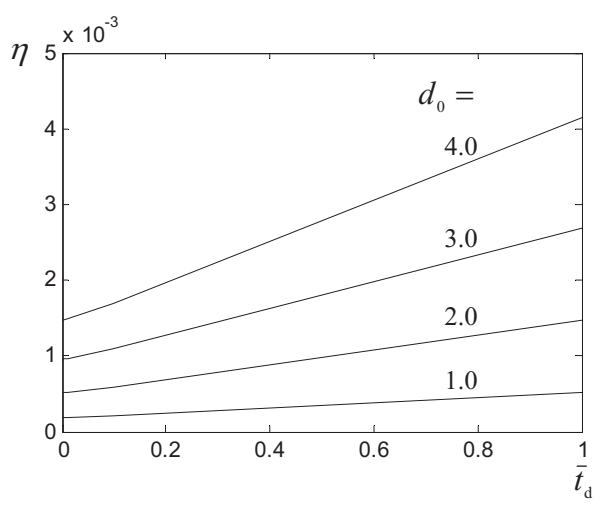

FIG. 9. The variation in $\eta\left(\mathrm{m}^{2} \mathrm{~J}^{-1 / 2} / \mathrm{A} \mathrm{s}\right)$ with $\bar{t}_{d}$ and $d_{0}(\mu \mathrm{m})$.

asperity height increases at constant $d_{0}$, the distance from the smooth plate to the bottom plate of asperities increases, which will lead to a higher pull-in voltage.

As it is found that the asperity height has a little effect within reasonable ranges of asperity radius and asperity height, we can neglect the effect of asperity on pull-in voltage, and use the approximation (18).

In expression (18), the value of $\eta$ is calculated for some common initial gap sizes of MEMS switch $d_{0}=1,2,3$, and $4 \mu \mathrm{m}, \bar{t}_{d}$ ranging from 0.0001 to 1 , and $\varepsilon_{0}=8.854$ $\times 10^{-12} \mathrm{C} / \mathrm{V} \mathrm{m}$. These results are plotted in Fig. 9, and it shows that depending on the design of the electrostatic domain, $\eta$ varies between $2 \times 10^{-4}$ and $5 \times 10^{-3} \mathrm{~m}^{2} \mathrm{~J}^{-1 / 2} / \mathrm{A} \mathrm{s}$ for typical dimensions of MEMS.

Therefore, the pull-in voltages $V_{\text {pull in }}$ in terms of $\bar{k}$ can be obtained from Eq. (18). Figure 10 provides the stiffness required to obtain a given pull-in voltage depending on the electric and geometric parameter $\eta$. It can be seen that in order to obtain a reasonable actuation voltage the spring constant has to stay below a limit depending on $\eta$.

\section{B. Pull out}

If the pull-out voltage exists, from the equilibrium equation (2), we can find that the electrostatic force should satisfy

$$
F_{E}=-\left.\left(F_{M}+F_{n}\right)\right|_{\delta \geqslant \delta_{1}}<0
$$

where $\delta_{1}$ is the critical approach at which pull out occurs in displacement control conditions, and which equals $\delta_{C}$ for $\lambda$

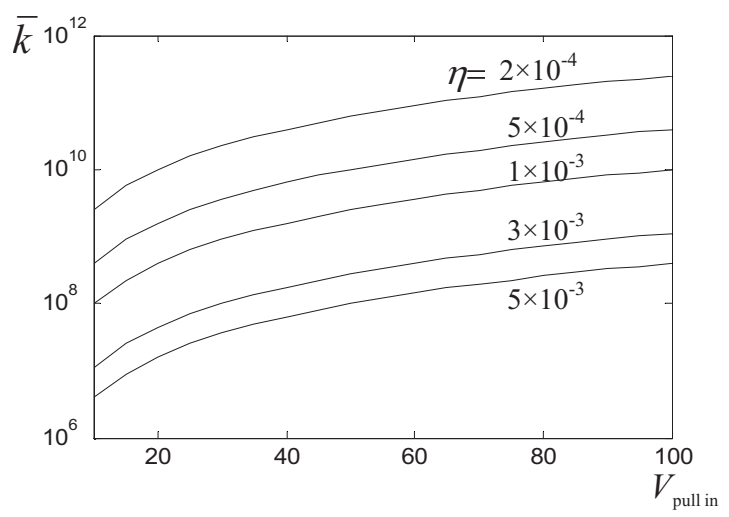

FIG. 10. Pull-in voltage (V) vs the normalized spring constant $\bar{k}\left(\mathrm{~N} / \mathrm{m}^{3}\right)$, the unit of $\eta$ is $\mathrm{m}^{2} \mathrm{~J}^{-1 / 2} / \mathrm{A} \mathrm{s}$. 


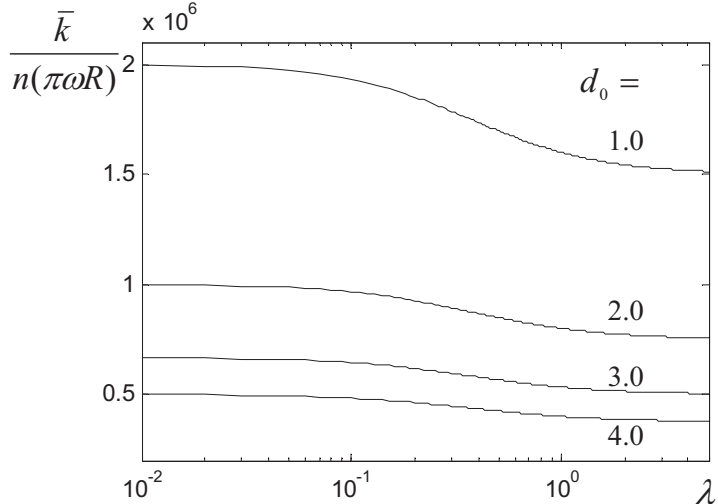

FIG. 11. Lower limit of the normalized equivalent spring constant for various initial gap sizes $d_{0}$ (unit in $\mu \mathrm{m}$ ) as a function of $\lambda$.

$>0.938$ and has been defined in Fig. 6. In the case of $\lambda$ $<0.938$, the critical approach is equal to $-z_{0}$. As the electrostatic force is attractive, Eq. (19) can be rewritten as

$$
k\left(d_{0}-d\right)>-F_{n}, \quad \delta \geqslant \delta_{1} .
$$

As for pull-in, we use $A_{a}$ to denote the apparent contact area (i.e., the electrode plate surface), the normalized spring constant is set to be $\bar{k}=k / A_{a}$. Hence, $\bar{k}$ represents the spring constant for a unit of apparent contact area, and $-F_{n} / A_{a}$ represents the total contact force (with adhesive contribution) on a unit of apparent contact area. If we assume that the number of asperities in contact on a unit of apparent contact area is $n$, then combining Eq. (20) with Eq. (10) yields

$$
\bar{k}\left(d_{0}-d\right)>-(\pi \varpi R) \sum_{i=1}^{n} \bar{F}_{n i}, \quad \delta \geqslant \delta_{1}
$$

where $\bar{F}_{n i}$ is the normalized contact force on $i$ th asperity. If we assume that all the asperities have the same height, the maximum normalized adhesive force is

$$
\max \left[-\sum_{i=1}^{n} \bar{F}_{n i}\right]=-n \cdot \bar{F}_{\mathrm{ad}},
$$

where $\bar{F}_{\text {ad }}$ is the maximum adhesive force on one asperity. In the general equation originally presented by Carpick et $a l .,{ }^{20,21}$ the Maugis' transition solution is approximated accurately for $0.1<\lambda<5$, and $\bar{F}_{\text {ad }}$ is expressed as a function of Maugis' transition parameter,

$$
\bar{F}_{\text {ad }}=-\frac{7}{4}+\frac{1}{4} \frac{\left(4.04 \lambda^{1.4}-1\right)}{\left(4.04 \lambda^{1.4}+1\right)} .
$$

Combining Eqs. (21)-(23), the lower limit of normalized equivalent spring constant, which avoids stiction is obtained, and presented in Fig. 11 for different initial gap size $d_{0}$. The mean separation $d$ in Eq. (21) is neglected for it is comparatively small to $d_{0}$ in contact configuration.
TABLE I. Parameters for electrostatic force.

\begin{tabular}{cccc}
\hline \hline$d_{0}$ & $t_{d}$ & $\varepsilon_{0}$ & $\varepsilon_{r}$ \\
\hline $2 \mu \mathrm{m}$ & $0.15 \mu \mathrm{m}$ & $8.85 \times 10^{-12} \mathrm{~F} / \mathrm{m}$ & 7.6 \\
\hline \hline
\end{tabular}

\section{Design example}

A typical material system in MEMS capacitive switches is chosen as a $1 \mathrm{D}$ design example. The material considered for the DC pads is aluminum, and a thin layer of $\mathrm{SiN}$ was deposited above the lower DC pad. ${ }^{22}$ The expected pull-in voltages $V_{\text {pull in }}$ is successively set up to be $20,40,60$, and $80 \mathrm{~V}$.

With the parameters reported in Table I, the upper limits of the spring constant, which allows for pull in to happen, can be computed using the framework previously presented in Sec. III A. Therefore, the lower limit of the spring constant, which allows avoiding stiction, can be computed from Eq. (21). This spring constant depends on the materials and on the surface finishing. Material parameters for $\mathrm{Al}$ and $\mathrm{SiN}$, and typical asperity radii are reported in Table II.

We assume a uniform distribution of identical asperities with a density of 100 asperities by $1 \mu \mathrm{m}^{2}$, which is in accordence to Ref. 22. The critical value of separation $z_{0}$ in Maugis theory equals $0.97 r_{0}$, where $r_{0}$ is the equilibrium separation of atoms for the Lennard-Jones potential. ${ }^{18}$ The value of $r_{0}$ is unknown for the materials in this design, but based on some relative data presented in Refs. 23-25, it is assumed that $z_{0}=0.35 \mathrm{~nm}$. In literature, work of adhesion $\varpi$ varies from the high value of $20 \mathrm{~mJ} / \mathrm{m}^{2}$, which is a representative value for oxide surface, to $0.01 \mathrm{~mJ} / \mathrm{m}^{2}$ for special antistiction surface coating. ${ }^{26}$ As this value is not well defined, a large range of values will be considered.

Figure 12 shows the upper and lower limits of the spring constant corresponding to different pull-in voltages versus the work of adhesion. From Fig. 12, it can be found that the proper value of equivalent spring constant can only be designed for low adhesion work, as for high work of adhesion range, the minimal spring constant avoiding stiction is larger than the maximal spring constant allowing for pull-in to happen.

In fact, this academic example is a crude simplification of reality, and the real problem is not as severe as what is shown in Fig. 12. For a real rough surface, the asperities do not have a constant height, and only a few of them will enter in contact. This motivates the discussions in Sec. IV

\section{STATISTICAL MODEL OF ROUGH SURFACE}

The contact of two rough surfaces is represented by the contact of a rough surface with a smooth plane. Indeed, based on the research works of Greenwood and

TABLE II. Parameters for contact.

\begin{tabular}{cccccr}
\hline \hline$R_{1}$ & $R_{2}$ & $E_{1}$ & $E_{2}$ & $\nu_{1}$ & $\nu_{2}$ \\
\hline $39.50 \mathrm{~nm}$ & $59.18 \mathrm{~nm}$ & $70 \mathrm{GPa}$ & $295 \mathrm{GPa}$ & 0.35 & 0.25 \\
\hline \hline
\end{tabular}




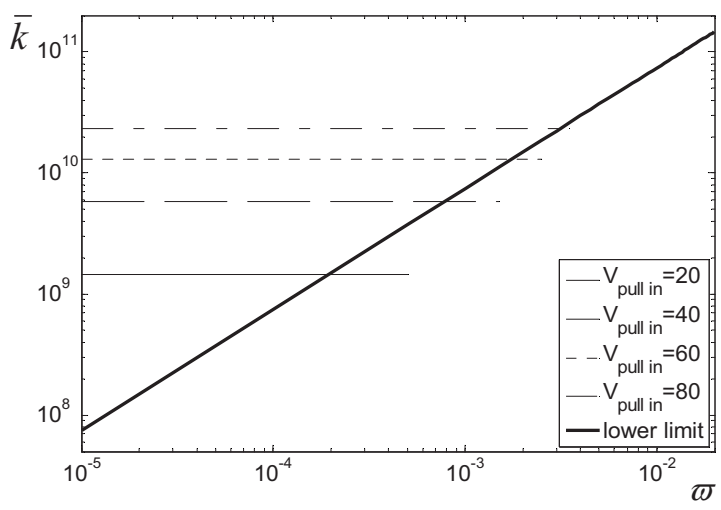

FIG. 12. Upper and lower limits of equivalent spring constant $\bar{k}\left(\mathrm{~N} / \mathrm{m}^{3}\right)$ vs the work of adhesion $\varpi\left(\mathrm{J} / \mathrm{m}^{2}\right)$ for different pull-in voltages $(\mathrm{V})$.

O'Callaghan, ${ }^{11,27,28}$ it can be concluded that the contact of two rough surfaces is negligibly different from the contact of a smooth and an equivalent rough surface.

Using the "asperity-based model" introduced by Greenwood and Williamson, ${ }^{11}$ the equivalent rough surface is described by a collection of spherical asperities with identical end radii, whose height $h$ has a statistical distribution, usually the Gaussian distribution

$$
\varphi(h)=\frac{1}{\sigma \sqrt{2 \pi}} \exp \left(\frac{-h^{2}}{2 \sigma^{2}}\right),
$$

where $\sigma=\sqrt{\sigma_{1}^{2}+\sigma_{2}^{2}}$ is the standard deviation in asperity height of the equivalent rough surface, and $\sigma_{1}$ and $\sigma_{2}$ are the standard deviations in asperity height of the two initial rough surfaces. The standard deviation in asperity height is also called the rms roughness of surface.

The distance $d$, which is defined as the separation between the two rough surfaces' mean planes of asperity heights, has to be replaced by the length from the equivalent rough surface's mean plane of asperity heights to the smooth surface (Fig. 13). Therefore, the number of asperities in contact per unit of surface is

$$
n=N \int_{d}^{\infty} \varphi(h) d h
$$

where the density of asperities $N$ is the number of asperities per unit area of surface. It should be noted that due to adhesion during the unloading process, asperities may remain in contact even if the height of asperity is lower than the equilibrium separation. Moreover, due to these adhesive forces the deformed position may be maintained within the distance $z_{0}$ in which adhesive forces are still acting. Therefore, the low limit of integration is set to $d+\delta_{c}$ in order to take into account this unloading effect (see Fig. 6), and Eq. (25) becomes

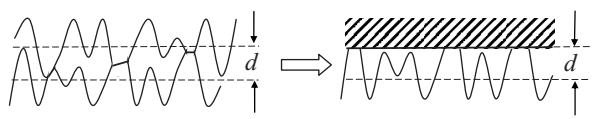

FIG. 13. Contact of two rough surfaces and the equivalent rough surface with smooth surface at a separation of $d$.

$$
n=N \int_{d+\delta_{C}}^{\infty} \varphi(h) d h,
$$

where $\delta_{c}$ is the critical approach at which abrupt pull out occurs for $\lambda>0.938$, according to Maugis' work. ${ }^{17}$ Kim's work $^{19}$ indicates that for low $\lambda$, the separation of the two surfaces occurs gradually and the abrupt pull-out takes place after the tensile force between the two surfaces has fallen to a low magnitude. From Kim's equations (13)-(15), it can be deduced that the critical value of separation $z_{0}$ in Dugdale approximation is the critical approach for low $\lambda$, and

$$
n=N \int_{d-z_{0}}^{\infty} \varphi(h) d h .
$$

Therefore, by combining Eqs. (26) and (27), the total contact force in Eq. (21) becomes

$$
\begin{aligned}
\sum_{i=1}^{n} \bar{F}_{n i} & =N \int_{\bar{d}_{+\Delta_{1}}}^{\infty} \bar{F}_{n}(\Delta) \varphi(\bar{h}) d \bar{h} \text { and } \Delta_{1} \\
& = \begin{cases}\Delta_{C}, & \lambda \geqslant 0.938 \\
-\bar{z}_{0} & \lambda<0.938,\end{cases}
\end{aligned}
$$

where any parameter with a superscript bar in the equation above is normalized with Eqs. (10) and (11), respectively. According to Kim's equation ((15)) and normalizing Eq. (11), as the dimensionless adhesive contact radius $C=0$ and $\xi=1$, it can be obtained

$$
\bar{z}_{0}=\frac{2}{\pi} \frac{1}{\lambda} .
$$

As the dimensionless approach (interference) $\Delta$ is equal to $(\bar{h}-\bar{d})$, Eq. (28) can be rewritten as

$$
\sum_{i=1}^{n} \bar{F}_{n i}=\frac{N}{\bar{\sigma} \sqrt{2 \pi}} \int_{\Delta_{1}}^{\infty} \bar{F}_{n}(\Delta) \exp \left[-\frac{(\Delta+\bar{d})^{2}}{2 \bar{\sigma}^{2}}\right] d \Delta,
$$

where $\bar{\sigma}$ equals the rms roughness $\sigma$ normalized by $\left(\pi^{2} \varpi^{2} R / K^{2}\right)^{1 / 3}$

The problem can now be solved similarly as what has been done for Eqs. (21) and (22) by using the expression (30) of the interaction forces: the critical problem is to obtain the maximum adhesive force of the rough surface with respect to $\bar{d}$. We note

$$
\bar{F}_{\max }=\max \left\{-\frac{N}{\bar{\sigma} \sqrt{2 \pi}} \int_{\Delta_{1}}^{\infty} \bar{F}_{n}(\Delta) \exp \left[-\frac{(\Delta+\bar{d})^{2}}{2 \bar{\sigma}^{2}}\right] d \Delta\right\} .
$$

The values of $\bar{F}_{\max }$ are computed as a function of the normalized surface roughness $\bar{\sigma}$ for different $\lambda$ $=0.1,0.3,0.5,1.0,5.0$. Results in terms of $\bar{F}_{\text {max }} /\left(N \bar{F}_{\text {ad }}\right)[\mathrm{see}$ Eq. (23)] are presented in Fig. 14. Figure 14 shows that the adhesive forces decrease rapidly with the increase of surface roughness $\bar{\sigma}$. For large value of $\lambda$, which physically corresponds to high adhesive work and soft materials (JKR regime), the decay rate of adhesive force is higher than for low $\lambda$ (DMT regime). This difference in the decay rates is attrib- 


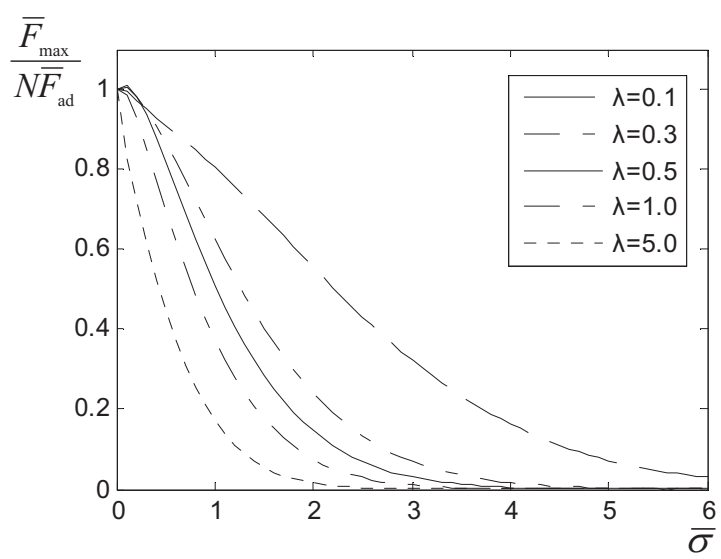

FIG. 14. The maximum adhesive force vs dimensionless rms surface roughness for different $\lambda$.

uted to the fact that DMT materials have long-ranged adhesive forces comparing to short-ranged adhesive forces of JKR materials.

It appears in Fig. 14 that the lower the $\lambda$, the higher the adhesive forces for a given rms roughness. The reason for this phenomenon is the use of normalized adhesive force. In fact, large $\lambda$ corresponds to the contact of compliant materials with high adhesive work, and this kind of contact naturally generates larger adhesive forces than the contact of hard materials with low adhesive work.

\section{DESIGN RESULTS FOR ROUGH SURFACE}

Let us consider the same design parameters than in the previous design example (Sec. III C), but with a nonuniform rough surface. The asperities have a constant radius of curvature, but their heights follow a Gaussian distribution. The density of asperity on the rough surface is kept equal to $100 / \mu \mathrm{m}^{2}$. A wide range of adhesive work is considered herein, the maximum value being $140 \mathrm{~mJ} / \mathrm{m}^{2}$, which corresponds to $\mathrm{Au}-\mathrm{Au}$ contact. ${ }^{25}$

Because the effect of asperities on pull-in voltage is neglected, the expected pull in voltages are still set up to be successively 20,40,60, and $80 \mathrm{~V}$, and the upper limits of the equivalent spring constant remain the same as in Sec. III C. In this previous design example, all the asperities had a constant height, which means the rms roughness of the surface was zero. For surface rms roughness equals $0.5,0.6,0.7$, and $1.0 \mathrm{~nm}$, successively, the lower limits of the equivalent spring constant required to avoid stiction were computed using the model presented in Sec. IV, and results are presented in Fig. 15 together with the upper limits.

From Fig. 15, it can be concluded that the rms roughness of the surfaces in contact has an important effect on the lower limits of the equivalent spring constant. With the increase in rms roughness, the design region becomes larger. Although the minimum equivalent spring constant avoiding stiction rises with increasing adhesive work, when the contact surfaces have a high rms roughness, a proper spring constant can still be found.

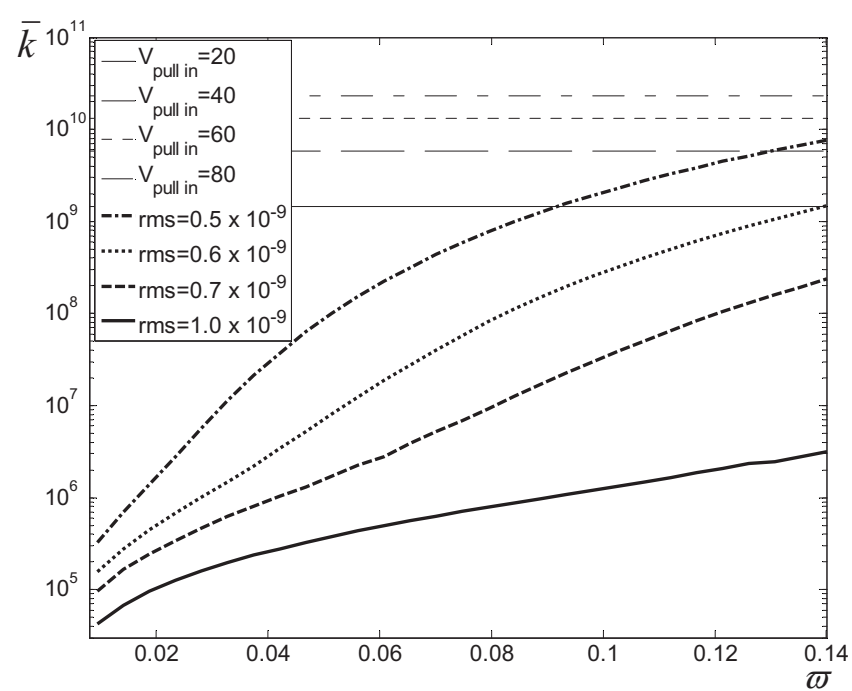

FIG. 15. Upper and lower limits of equivalent spring constant $\bar{k}\left(\mathrm{~N} / \mathrm{m}^{3}\right)$ vs the work of adhesion $\varpi\left(\mathrm{J} / \mathrm{m}^{2}\right)$ for different pull-in voltages $(V)$ and $\mathrm{rms}$ roughness $(m)$.

\section{PARAMETER IDENTIFICATION}

In Secs. II and IV numerous parameters were considered, and their identification is crucial to the application of the presented models. Generally, the parameters involved can be categorized into four basic groups: electrical parameters, mechanical properties, adhesive parameters, and surface properties.

\section{A. Electrical parameters}

The main electrical parameter used in this model is the permittivity of dielectric material, which can be found in handbooks or measured using some common methods.

\section{B. Mechanical properties}

A significant amount of research has been carried out to measure the mechanical properties of materials at the microscale. ${ }^{29-32}$ The value of Young's modulus $E$ can be measured by nanoindentation or micro tensile-test.

For the common structure materials of MEMS, polysilicon has reported values of the modulus $E$ ranging from 135 to $173 \mathrm{GPa}$ and a value of 0.23 has been found for Poisson's ratio $\nu{ }^{30,33}$ Young's modulus of single-crystal silicon ranges from 130 to $178.6 \mathrm{GPa}$ for the different crystalline directions with a Poisson's ratio $\nu=0.064$ for $\mathrm{Si}\langle 100\rangle$ and 0.279 for $\mathrm{Si}\langle 110\rangle .^{34,35}$ Young's modulus of gold ranges from 43 to $117 \mathrm{GPa}$, depending on the crystalline direction with a Poisson's ratio $\nu=0.42 .{ }^{36}$ A typical value of $78 \mathrm{GPa}$ for the elastic modulus of bulk gold has been used in Refs. 37 and 38. Silicon nitride has a Young's modulus $E$ ranging from 220 to $295 \mathrm{GPa}$ and a Poisson's ratio $\nu$ around 0.26 . $^{22,39,40}$ The mechanical properties for other materials can be found in Refs. 29 and 39. 


\section{Adhesive parameters}

Generally, the work of adhesion $\varpi$ ranges for $1 \mathrm{~J} \mathrm{~m}^{-2}$ for metallic materials to $10 \mathrm{~mJ} \mathrm{~m}^{-2}$ for molecular solids. ${ }^{41}$ The measurement of the work of adhesion can be achieved at two scales: the micro- and nanoscales. ${ }^{42,43}$.

At microscale, ${ }^{8}$ by measuring the sticking part of a cantilever beam contacting a substrate, the work of adhesion can be estimated. However, this technique is very sensitive to the surface roughness of the beam and of the substrate, which means the effect of surface roughness has already been included.

As, in our applications, the contact of asperities is studied, the measurement at nanoscale is required in order to avoid this roughness dependency. This can be achieved by using an atomic force microscope (AFM) which can accurately and precisely measure the adhesion force of a pointto-point contact. The AFM is composed of a cantilever beam at the end of which a tip is attached. To measure the work of adhesion between two materials, this tip is coated with one of them, while the test specimen is made of the second one. When moving the tip toward the specimen, at some point the attractive forces will be such than the specimen would grab the tip. Then, a force-displacement curve is obtained when reversing the tip motion to separate the materials. ${ }^{44}$ This process allows measuring the pull-out force (maximum adhesive force) between the AFM tip coating and the substrate surface. The work of adhesion can be calculated from the corresponding adhesive contact theory. Details of the work of adhesion measurement techniques can be found in Ref. 43 .

In the definition of Dugdale assumption, the maximum adhesive traction $\sigma_{0}$, which satisfies $\varpi=\sigma_{0} \cdot z_{0}$ (Fig. 4), can be determined by the expression of Lennard-Jones potential. $^{41}$ The maximum value is given by $\sigma_{0}$ $\approx 1.0264 \approx / r_{0},{ }^{41}$ where $r_{0}$ is the equilibrium distance of atoms/molecules in Lennard-Jones potential theory. Then, the critical value of separation in Dugdale assumption $z_{0}$ $=0.97 r_{0}$ can be directly obtained.

For gold, the equilibrium interatomic distance $r_{0}$ was assumed equal to $0.3 \mathrm{~nm}$ in Ref. 45. Ruggiero and Paolo ${ }^{41}$ applied $0.1 \mathrm{~nm}$ as the common value of $r_{0}$. A typically value $r_{0} \approx 0.28 \mathrm{~nm}$ was used for metals in Ref. 46. For molecular solids, $r_{0}$, which is the equilibrium intermolecular distance, was about $0.3-0.5 \mathrm{~nm}^{47}$

\section{Surface properties}

The surface properties involved in the model are the rms roughness $\sigma$, the assumed radius of the spherical asperities $R$, and the asperities' density $D$. All these values can be obtained through three dimensional topographic measurements of the surface using AFM. In MEMS, the surface properties do not only depend on the material of the surface but they also depend on the microfabrication processes and treatment conditions. Using AFM, Yu and Liu measured the surfaces of microcapacitive switches, ${ }^{22}$ for a $0.15 \mu \mathrm{m} \mathrm{SiN}$ layer deposited on $\mathrm{Ti}, \mathrm{Cr}, \mathrm{Al}$, and $\mathrm{Cu}$ thin films respectively, the rms roughness $\sigma$ was found to range from 2.19 to $14.81 \mathrm{~nm}$, asperities radius $R$ from 31.11 to $63.22 \mathrm{~nm}$, and the asperities' density $D$ from
158 to $41 \mu \mathrm{m}^{-2}$. rms roughnesses of 0.6 and $0.8 \mathrm{~nm}$ were reported for treated and untreated silicon nitride layers respectively in Ref. 48. The rms roughness of polysilicon layer varies from about 1 to $13.6 \mathrm{~nm}$, depending on micromachining techniques and treatment conditions, as studied in Refs. 9, 48, and 49. Typical value for the polysilicon asperities radius of $R=220 \mathrm{~nm}$ was used in Ref. 50. The surface roughness of the silicon wafer ranges from 0.1 to $1.0 \mathrm{~nm}$, depending on the surface modification process, while the radius of asperity $R$ varies from 1.8 to $76 \mu \mathrm{m}$ and the asperities' density $D$ varies from 4.4 to $38.4 \mu \mathrm{m}^{-1}$. $^{4,51}$ In RF MEMS switches, the measured rms roughnesses of $\mathrm{Au}$ films were measured as being 3.6 and $6.9 \mathrm{~nm}$ on the beam and on the contactor, respectively, ${ }^{52}$ Gregori $^{45}$ evaluated the rms roughnesses to $3.5 \mathrm{~nm}$ with an asperity's radius of $120 \mathrm{~nm}$ for the $\mathrm{Au}$ films of a new switch, while he evaluated the rms roughnesses to $2 \mathrm{~nm}$ with asperity's radius of $270 \mathrm{~nm}$ for a used one.

\section{CONCLUSIONS}

An analytical design method for the equivalent spring constant in MEMS switches is presented here. A proper design region of the equivalent spring constant is defined. The upper limit of spring constant is set to obtain a reasonable actuation (pull in) voltage, and the lower limit of spring constant is set to avoid in-use stiction, which means the restoring elastic forces are high enough for snap back to happen after pull in.

The upper limit depends mainly on the expected actuator voltage and on the geometric parameters, such as initial gap size and thickness of dielectric layer. The surface roughness has little effect on the upper limit.

The adhesive work of contact surfaces and surfaces' roughness are the main factors of adhesive force, which is the crucial factor of the lower limit. High adhesive work and low surface roughness lead to high adhesive forces, which in turn requires a high equivalent spring constant to avoid inuse stiction.

For a given actuation voltage, it might be impossible to find an adequate spring constant in between the 2 limits. The adhesive forces can be reduced by choosing the contact materials with low surface adhesive work and by reducing the real contact area. From Fig. 15, it can be seen that the decrease of the adhesive work is not drastic enough to reduce the lower limit of the design region. Therefore reduction of the real contact area is mandatory. This can be achieved by increasing the surface rms roughness. The design region of equivalent spring constant can also be enlarged by using larger electrodes area for electrostatic forces and smaller electrodes area for contact.

In the current work, other kinds of adhesion forces, such as capillary forces, electrostatic forces from dielectric charging, are not considered, and the plastic deformation of asperities are neglected, but these effects will be considered in future work. 


\section{ACKNOWLEDGMENTS}

The work of the first author has been supported by the project COROMIS, "First Postdoc Project 2007" funded by the Walloon Region of Belgium. The second author acknowledges the financial support of the Belgian National Fund for Scientific Research.

${ }^{1}$ V. Rochus, D. J. Rixen, and J.-C. Golinval, Int. J. Numer. Methods Eng. 65, 461 (2006).

${ }^{2}$ V. Rochus, Sens. Lett. 6, 88 (2008).

${ }^{3}$ C. H. Mastrangelo, Tribol. Lett. 3, 223 (1997).

${ }^{4}$ Z. Rymuza, Microsyst. Technol. 5, 173 (1999).

${ }^{5}$ R. Maboudian and R. T. Howe, J. Vac. Sci. Technol. B 15, 1 (1997).

${ }^{6}$ N. Tas, T. Sonnenberg, H. Jansen, R. Legtenberg, and M. Elwenspoek, J. Micromech. Microeng. 6, 385 (1996), and references therein.

${ }^{7}$ R. Maboudian, Surf. Sci. Rep. 30, 207 (1998).

${ }^{8}$ W. Merlijn van Spengen, R. Puers, and I. De Wolf, J. Micromech. Microeng. 12, 702 (2002).

${ }^{9}$ A. Hariri, J. W. Zu, and R. Ben Mrad, J. Micromech. Microeng. 16, 1195 (2006).

${ }^{10}$ A. Lumbantobing, L. Kogut, and K. Komvopoulos, J. Microelectromech. Syst. 13, 977 (2004).

${ }^{11}$ J. A. Greenwood and J. B. P. Williamson, Proc. R. Soc. London, Ser. A 295, 300 (1966).

${ }^{12}$ K. L. Johnson, K. Kendall, and A. D. Roberts, Proc. R. Soc. London, Ser. A 324, 301 (1971).

${ }^{13}$ B. V. Derjaguin, V. M. Muller, and Y. P. Toporov, J. Colloid Interface Sci. 53, 314 (1975).

${ }^{14}$ D. Maugis, Langmuir 11, 679 (1995).

${ }^{15}$ M. Zahn, Electromagnetic Field Theory: A Problem Solving Approach (Wiley, New York, 1979).

${ }^{16}$ E. Barthel, J. Colloid Interface Sci. 200, 7 (1998).

${ }^{17}$ D. Maugis, J. Colloid Interface Sci. 150, 243 (1992).

${ }^{18}$ K. L. Johnson and J. A. Greenwood, J. Colloid Interface Sci. 192, 326 (1997).

${ }^{19}$ K. S. Kim, R. M. McMeeking, and K. L. Johnson, J. Mech. Phys. Solids 46, 243 (1998).

${ }^{20}$ R. W. Carpick, D. F. Ogletree, and M. Salmeron, J. Colloid Interface Sci. 211, 395 (1999).

${ }^{21}$ O. Piétrement and M. Troyon, J. Colloid Interface Sci. 226, 166 (2000).

${ }^{22}$ A. B. Yu, A. Q. Liu, Q. X. Zhang, and H. M. Hosseini, J. Micromech. Microeng. 16, 2157 (2006).

${ }^{23}$ T. Lin, X. F. Bian, and J. Jiang, Phys. Lett. A 353, 497 (2006).

${ }^{24}$ M. P. Allen and D. J. Tildesley, Computer Simulation of Liquids, Clarendon Press, Oxford Science Publications p. 22 (1989).

${ }^{25}$ Z. Zong, Y. F. Cao, N. Rahbar, and W. Soboyejo, J. Appl. Phys. 100,
104313 (2006).

${ }^{26}$ W. R. Ashurst, C. Yau, C. Carraro, R. Maboudian, and M. T. Dugger, J. Microelectromech. Syst. 10, 41 (2001).

${ }^{27}$ J. A. Greenwood and J. H. Tripp, Proc. Inst. Mech. Eng. 185, 625 (1971).

${ }^{28}$ M. O'Callaghan and M. A. Cameron, Wear 36, 79 (1976).

${ }^{29}$ H. Lee, R. A. Coutu, S. Mall, and K. Leedy, J. Micromech. Microeng. 16, 557 (2006).

${ }^{30}$ W. N. Sharpe, K. M. Jackson, K. J. Hemker, and Z. Xie, J. Microelectromech. Syst. 10, 317 (2001).

${ }^{31}$ K. Sato, M. Shikida, T. Yoshioka, T. Ando, and T. Kawabata, Solid State Sensors and Actuators, IEEE Transducers '97, Chicago, IL, Vol. 1, p. 595 (1997)

${ }^{32}$ X. D. Li, B. Bhushana, and K. Takashimab, Ultramicroscopy 97, 481 (2003).

${ }^{33}$ J. W. Wittwer, M. S. Baker, and L. L. Howell, J. Microelectromech. Syst. 15, 33 (2006).

${ }^{34}$ C. Wilson and P. Beck, J. Microelectromech. Syst. 5, 142 (1996).

${ }^{35}$ Y. Matsuoka, Y. Yamamoto, K. Yamada, S. Shimada, M. Tanabe, A. Yasukawa, and H. Matsuzaka, J. Micromech. Microeng. 5, 25 (1995).

${ }^{36}$ G. Rubio, N. Agraït, and S. Vieira, Phys. Rev. Lett. 76, 2302 (1996).

${ }^{37}$ J. H. Kim, D. J. Srolovitz, and P.-R. Cha , J. Appl. Phys. 100, 054502 (2006).

${ }^{38}$ J. W. Tringea and T. A. Uhlman, J. Appl. Phys. 93, 4661 (2003).

${ }^{39} \mathrm{X}$. Weihua, "Mechanical properties of materials at micro/nano scales," Ph.D. thesis Hong Kong University, 2003.

${ }^{40}$ H.-J. Butt, M. Farshchi-Tabrizi, and M. Kappl, J. Appl. Phys. 100, 024312 (2006).

${ }^{41}$ R. Gissi and P. Decuzzi, J. Appl. Phys. 98, 014310 (2005).

${ }^{42}$ M. P. de Boer and T. A. Michalske, J. Appl. Phys. 86, 817 (1999).

${ }^{43}$ H.-J. Butt, B. Cappella, and M. Kappl, Surf. Sci. Rep. 59, 1 (2005).

${ }^{44}$ B. Stegemann, H. Backhaus, H. Kloss, and E. Santner, in Modern Research and Educational Topics in Microscopy, edited by A. Méndez-Vilas and J. Diaz (Formatex, Badajoz, Spain, 2007).

${ }^{45}$ G. Gregori and D. R. Clarke, J. Appl. Phys. 100, 094904 (2006).

${ }^{46}$ D. Erts, A. Lohmus, R. Lohmus, H. Olin, A. V. Pokropivny, L. Ryen, and K. Svensson, Appl. Surf. Sci. 188, 460 (2002).

${ }^{47}$ P. Sahoo and A. Banerjee, J. Phys. D 38, 4096 (2005).

${ }^{48}$ E. J. Thoreson, "From nanoscale to macroscale, using the atomic force microscope to quantify the role of few-asperity contacts in adhesion," Ph.D. thesis Worcester Polytechnic Institute, 2006.

${ }^{49}$ C. K. Boraa, E. E. Flater, M. D. Street, J. M. Redmond, M. J. Starr, R. W. Carpick, and M. E. Plesha, Tribol. Lett. 19, 37 (2005).

${ }^{50}$ F. W. DelRio, M. L. Dunnb, and M. P. de Boer, Scr. Mater. 59, 916 (2008).

${ }^{51}$ C. Gui, M. Elwenspoek, N. Tas, and J. G. E. Gardeniers, J. Appl. Phys. 85, 7448 (1999).

${ }^{52}$ H. Kwon, S.-S. Jang, Y.-H. Park, Y.-D. Kim, H.-J. Nam, and Y.-C. Joo, J. Micromech. Microeng. 18, 105010 (2008). 